\title{
Un acercamiento teórico del desempeño laboral
}

A theoretical approach to job performance

Ticahuanca Sandoval Fernando Irving a(i)*, Mamani Palacios Bryan Rogger b(i)

a,b Universidad Peruana Unión, Lima, Perú

\section{Resumen}

La finalidad, de este estudio es desarrollar una aproximación teórica breve del desempeño laboral mediante su origen, modelo teórico y teoría de sus dimensiones. Los inicios y desarrollo del desempeño laboral se sustentan en el devenir conceptual a través de las etapas secuenciales relacionadas a la motivación y complacencia del empleado, orientación hacia el consumidor y estrategias. Uno de los modelos teóricos afirma que el desempeño pugna un rol determinante en la función del uso del conocimiento que hace cada sujeto. El desempeño laboral se concibe como un compuesto de actividades y el proceder observado en los trabajadores los cuales afectan a la finalidad de la organización. Además, sus dimensiones como desarrollo de la actividad principal, permite el rendimiento en las tareas, comportamiento contraproducente y rendimiento en el contexto de los trabajadores como retribución óptima para la organización; la contratación y retención de los empleados es una actividad para contratar a los mejores profesionales y por ende crear estrategias de retención mediante reconocimientos; educación laboral; y ambiente laboral. De esa manera este breve trabajo teórico es relevante porque permite esclarecer conceptualmente el desempeño laboral desde sus inicios, así como el fundamento teórico que permitirá realizar otros tratamientos teóricos del desempeño laboral.

Palabras Clave: Desempeño laboral; desarrollo; retención; educación laboral; ambiente laboral

\section{Abstract}

The purpose of this study is to develop a brief theoretical approach to job performance through its origin, theoretical model and theory of its dimensions. The beginnings and development of job performance are supported by the conceptual evolution through sequential stages related to employee motivation and satisfaction, consumer orientation and strategies. One of the theoretical models affirms that performance plays a determining role in the function of the use

\footnotetext{
* Autor de correspondencia:

E-mail: fernandoticahuanca@upeu.edu.pe

DOI: https://doi.org/10.17162/riva.v8i1.1633

Recibido: 14/10/2021 Aceptado: 8/12/2021
} 
of knowledge made by each subject. Job performance is conceived as a composite of activities and behavior observed in workers which affect the organization's purpose. In addition, its dimensions as development of the main activity, allows performance in tasks, counterproductive behavior and performance in the context of workers as optimal retribution for the organization; recruitment and retention of employees is an activity to hire the best professionals and thus create retention strategies through recognition; labor education; and work environment. Thus, this brief theoretical work is relevant because it allows to clarify conceptually the job performance from its beginnings, as well as the theoretical foundation that will allow to carry out other theoretical treatments of job performance.

Keywords: Job performance; developing; retention; job education; work environment

\section{Introducción}

Hoy en día las organizaciones están cambiando con mayor celeridad. En el entorno laboral existe una gran demanda de expertos con habilidades sensibles aptos para acomodarse o adecuarse a las continuas versatilidades de las empresa (Vercelli \& Thomas, 2008), consecuentemente las emociones de los empleados contribuyen en su rendimiento (Rosas-reyes \& Roberto, 2015).

A nivel global, las entidades están en constante enfrentamiento de suplir la necesidad de enriquecer el desarrollo laboral de su empresa, así confortar y sostenerse de la mejor forma dentro del mercado laboral (Araujo \& Leal, 2007). El desempeño laboral no solo se centra al mayor rendimiento obtenido, puesto ,que, se puede comprender que su campo se extiende al gozo de los individuos en su espacio de quehacer (María \& Pérez. 2011), sino se toma en consideración dichas causas las entidades que no logren tener el control de su existencia emocional, tendrán como consecuencia diversas luchas internas que afecten su eficiencia y desarrollo (Sanders et al. 2008).

Entender la inteligencia emocional es significativo, pues de él acatan otros individuos que son influenciados por la utilización de la misma, perjudicando el desempeño de toda la organización (Bautista et al., 2020). Debido que es un diseño para conseguir una considerable eficiencia, productividad y competitividad (Mamanil \& Avendaño, 2019).

En cambio, respecto al desempeño laboral se han realizado algunos tratamientos teóricos, así como investigaciones empericas(Baltodano \& Leyva, 2020), que presentan la trascendencia del constructo, identificando modelos como el de Cogollo quien afirma que la falta de condiciones laborales tiene un dominio vital en cuanto al ejercicio técnico en los diferentes ámbitos de desempeño; como también los modelos de Berón quien determina la trascendencia que tiene el exceso laboral por diversas funciones, demasiada responsabilidad, 
carencia de personal en la vigilancia, fallas en la comunicación de grupo de trabajo, establecimiento de turnos y la carga emocional (Lastini \& Veliz, 2016), dichas que son directamente proporcional al resultado final de desempeño laboral en las empresas (Aguirre, 1991). Espinoza et al. (2015), presenta una evolución del término desempeño, identificando modelos como el de Álvarez quien afirma que desempeño tiene una labor precisa en la utilización del conocimiento que hace cada persona, también propone que es notable al referirnos en cuanto al desempeño, eludir el rompimiento de los aspectos volitivos, cognitivos y afectivos; menciona a Ribes, Perelló, \& Herrero (2018) quien asocia el valor de autoevaluación, perfeccionamiento y excelencia.

En los últimos estudios de Torres et al. (2021), se ha encontrado que la inteligencia emocional tiene un angosto vínculo con el desempeño laboral de los técnicos de la salud debido ,que, para alcanzar una buena expansión de sus funciones y poder cumplir con las metas.

Álvarez, Porraspita, Indacochea (2018) resalta una perspectiva del término desempeño, identificando modelos como el de Koopmans quien afirma que el desempeño tiene un rotundo rol en la ampliación del conocimiento que compone cada persona, además propone que es vital al referirnos al desempeño evitando el desprendimiento de las composturas cognitivas. Por su parte, (Baltodano \& Leyva, 2020)realiza una presentación evolutiva del término, identificando modelos como el de cogollo quien afirma que la falta de condiciones laborales tiene una influencia vital respecto al ejercicio profesional en los diferentes ámbitos de desempeño, como también los modelos de Argoti (2020) quien determina la relevancia que estos tienen y son rectamente proporcionales al producto final del desempeño en las empresas. En tanto, Chavez (2020) realiza un estudio enfocado al capital intelectual, se trata de un estudio en el que se incentiva a valorar el esfuerzo del empleado y en el cual la autora incentiva a la empresa a incrementar la capacidad remunerativa para la aun mayor mejoría del rendimiento laboral.

La presente investigación tiene el propósito de explicar teóricamente el desempeño laboral mediante su origen y modelo teórico. Así también mediante el tratamiento teórico y sus dimensiones; segmentado en tres dimensiones (Desempeño en las tareas, desempeño en el contexto y comportamiento contraproducente) (Koopmans, 2015), las cuales son quien destaca la importancia del aporte de los trabajadores en la empresa según su respectivo margen de capacidad productiva en el cual se mencionan también sugerencias para incentivar la competitividad en el ambiente laboral; todo esto después de haber tenido la muestra respectiva de la población encuestada. 
También obtuvimos información respecto al aporte teórico de otros autores que mencionan conceptos importantes enfocados en el desempeño laboral en la base de datos de información científica, como sciencedirect, Redalyc, scielo, google scholar, dialnet y repositorios; toda esta información será almacenada y procesada para finalmente realizar las citaciones con sus respectivas referencias bibliográficas en el gestor de información Mendeley.

\section{Desarrollo o Revisión}

\subsection{Origen teórico del desempeño laboral}

En los posteriores periodos con relación al, desempeño laboral, las teóricas se han desarrollado facilitando cuantioso alcance a la inteligencia humana que es un eje distinguido de las entidades, que, es él ser. En el momento que se expone al desempeño gremial se establece con la estimación del desempeño motivo que está conectado rectamente un individuo con otro y cómo van progresando estos criterios.

Medina (2017) menciona que a mediados de la época media empleaban un procedimiento de cualificación que eran utilizados por los jefes, para ellos como para sus subalternos y de ese sistema se desenvolvía la estimación del desempeño, siguiendo (Lastini \& Veliz, 2016) la primera serie se usó una empresa de fábrica textil (Wolfe et al., 1990), la cual se basaba en la productividad frecuente de los trabajadores, (Topa \& Morales, 2006), mediante la retribución de un presente libro a cada trabajador y donde el inspector registraba el desempeño cotidiano calculando la clasificación por colores que señalaban los niveles de utilidad.

Tiempo después Drucker (1999), inicio la revisión de nuevas concepciones acerca del desempeño laboral, sugiere tener que asignar frescas explicaciones de este vocablo, manifestando medidas innovadoras, Chiavenato (2002) expone que el desempeño es la capacidad, que tiene el personal de lograr dentro de su espacio de trabajo, la cual obedece a múltiples factores que aplica un individuo y manifieste su trabajo (Newstrom, 2000), propone el interés de la fijación de objetivos, estimulándose de esta forma los comportamientos y habilidades (Robbins, y Judge, 2009), que se encadena con las habilidades, enunciando que en el tamaño en la que el empleado aumente sus idoneidades, entonces su función también mejorara (Benavidez Espíndola, 2002).

\subsection{Origen Modelo teórico de desempeño laboral de Koopmans}

El modelo de desempeño laboral de Koopmans (2015) se realizó mediante estadísticos descriptivos, modelos correlacionales y exploratorios. Según los resultados el instrumento realizado y validado, observamos que se tiene una estructura tridimensional; 
debido a que conlleva bastante similitud con cada una de las dimensiones del desempeño laboral; el cual también lleva un grado de coincidencia en el factor personalidad con otros instrumentos, pero un grado de confiabilidad muy superior a diferencia de otras investigaciones.

Koopmans (2015) lo define como la variable dependiente fundamental en lo que respecta a el área de RRHH, (Rodríguez-Marulanda \& Lechuga-Cardozo, 2020) donde también se puede mostrar un enfoque de acuerdo con criterios sistémicos, por ello se transmite un nivel de competencia constante en el ámbito laboral con el propósito de fomentar la auto exigencia, lo cual demuestra el grado de ejecución de los trabajadores en la empresa a un plazo determinado, con el debido esfuerzo dan a conocer al entorno social como también entorno laboral, sus respectivas cualidades y competencias que son difíciles de reemplazar (Ana et al., 2011; Hernández, 2008).

Según Koopmans (2015) el desempeño laboral es un conjunto de comportamientos y actitudes observados en los colaboradores los cuales intervienen con los objetivos de la empresa(Salanova et al., 2014), ya que es el valor que se espera aportar a la empresa de las distintos aspectos conductuales que una persona lleva acabo en un en el periodo laboral (Pastor, 2018), como también el ranking de empleados varían según su desempeño como tales (María \& Pérez, 2011), estas acciones son medibles, ordenadas y rigurosas; ya que de esta forma no remunerativa también se incentiva al trabajador a sentirse cómodo en la empresa (Ortega, 2015).

\subsection{Desempeño Laboral}

Se le denomina desempeño laboral a el conjunto de acciones observadas por el empleado (Koopmans, 2015), desde la perspectiva de Argoti (2020), es el valor que cada individuo llega a sumar en la empresa de manera individual de muchas formas posibles en un determinado lapso de tiempo, en la búsqueda de los objetivos fijados, de tal manera que se pueda ejecutar la medición y rendimiento del colaborador (Aguirre, 1991).

Según Robbins y Judge (2013), aporta con el estudio en mención del desempeño como uno de los pilares fundamentales de la psicología del desempeño, el cual va enfocado principalmente hacia la obtención de un final consciente, pero en este también intervienen otros aspectos con la capacidad de facilitar o entorpecer esta concordancia entre comportamiento y resultado.

\subsubsection{Desempeño en las tareas.}

Eufracio (2018), el desempeño laboral en una empresa es directamente proporcional hacia la productividad, al respecto Bolzan (2018) lo denomina como el rendimiento de uno o 
más de dos trabajadores respecto a las funciones en las que se propone el cumplimiento de las metas y los objetivos establecidos en una organización. Por su parte, Ribes, Perelló, \& Herrero (2018) señalan que calidad de labor que realiza un empleado o trabajador en su respectiva área o tarea, mediante el fortalecimiento de sus habilidades y competencias personales, lo cual conlleva hacia los resultados establecidos por la organización (Martín, 2018).

Por lo tanto, el desempeño laboral como el conjunto de acciones que se realizan de manera eficiente respecto al capital humano en cumplimiento con las metas establecidas por la empresa, la cual cumple un rol importante en función a sus capacidades, habilidades y destrezas laborales. En ese sentido, Ivars et al. (2016) resalta un factor importante en la determinación de grupos de trabajo en la empresa, también(Forbes, 2013), y así desarrollarse de manera eficiente en su área de trabajo, la cual abarca el compromiso de la organización o institución (Pedraza et al., 2010), la cual está relacionada con los objetivos y metas alcanzados en las actividades, debido a esto es de suma importancia mantener una adecuada, como también utilizar las técnicas y métodos necesarias para representar las actividades desarrolladas (Fuentes et al., 2019), considerando que la formación es la actualización y capacitación constante de las áreas de la empresa, con el fin de generar una organización sumamente competitiva.

\subsubsection{Desempeño en el contexto.}

Rodríguez-Marulanda \& Lechuga-Cardozo (2020) describe a el desempeño laboral como la efectividad, en función a criterios sistémicos, por lo cual se mide de acuerdo a la capacidad de los dirigentes para adaptarse y crecer, además Hernández (2008) resalta el enfoque de ejecución alcanzado por el empleado en el logro de los objetivos dentro de la empresa en un tiempo establecido (Ana et al., 2011)que existan empleados con alto desempeño o servidores con cualidades y competencias que son difíciles de reemplazar

Según Chiavenato (2009) el desempeño laboral es un conjuntos de metas observadas en los trabajadores, desde la perspectiva de Palací (2005), es el valor que se espera aportar a la empresa de los diferentes desempeños conductuales que una persona lleva a cabo en un lapso de tiempo, en la búsqueda de objetivos fijados.

Según Robbins y Judge (2009), quien realiza un estudio empírico respecto al desempeño y sus previos resultados, resalta el hecho de buscar un propósito consciente, pero en él también intervienen otros agentes con la capacidad de facilitar esta asociación entre resultado y comportamiento (Mejía, 2010). 


\subsubsection{Comportamiento contraproducente}

Respecto al comportamiento contraproducente se puede confirmar que se dice que no solo reconoce los sentimientos propios, sino que también los ajenos, (Pacheco \& Fernández-berrocal, 2004) para poder auto-motivarse y también motivar a los demás(Forbes, 2013), mejorando positivamente las emociones internas y las relaciones con el entorno laboral (Navarro et al., 2016) demostrando interés y preocupación por todos los aspectos psicológicos (Moscoso, 2019).

Según Buitrón \& Navarrete (2008) es una habilidad para percibir, reconocer y valorar las emociones, como también para expresar y regular la opinión de cada individuo, (Hernández \& Vázquez, 2018) ya que es la capacidad de expresar nuestras emociones y comprenderlas como habilidad intrínseca del individuo (Sladogna, 2017) también como un recurso personal que facilitaría una mejor percepción de auto-eficacia y autovaloración (Araujo \& Leal, 2007) ya que nos concede la destreza de conocer nuestros propios sentimientos y ser eficaces en nuestros deberes.

\section{Tabla 1}

Resumen de las dimensiones del Desempeño Laboral

\begin{tabular}{ll}
\hline Dimensiones & \multicolumn{1}{c}{ Resumen } \\
\hline & El desempeño en las tareas significa cumplir con las tareas y \\
& responsabilidades en un trabajo (Wu \& Liu, 2006). \\
& Clegg \& Cooper (2009) también, aportan, directa o \\
& indirectamente al centro de la organización. \\
& Koopmans et al. (2011) manifiestan que todas las actividades, \\
Desempeño de & se, fraccionan en, dos tipos de conductas, la, primera, que, \\
tareas & está asociado a la serie de transformación de los recursos en \\
& servicios propios de la organización, mientras, el otro tipo de \\
& conductas comprenden aquellas actividades que actúan como \\
& soporte a la esencia profesional. \\
& En consecuencia, el desempeño de la tarea engloba, \\
& conductas, direccionadas a la fabricación de bienes o \\
& servicios (Rotundo, 2002). \\
\hline Desempeño & Para Beverly et al. (2012) el desempeño contextual como \\
& conductas, espontáneas, y personales por parte de los \\
& empleados, estos rebasan a los esperados para su sitio de \\
& trabajo (Álamo \& Marquez, 2012), en consecuencia, estos \\
\hline
\end{tabular}




\begin{tabular}{ll}
\hline & comportamientos, impulsan, un, mejor movimiento de la \\
& entidad. (Pegoraro, 2009). \\
& Tratar el comportamiento contraproducente como un \\
& comportamiento intencional que daña intencionalmente a las \\
& organizaciones y a las personas, esto constituye una \\
& exhortación para el bienestar de la organización y los \\
& empleados (Omar, 2010). Omar et al. (2012), dicen, que, son \\
& comportamientos torcidos, es decir, son comportamientos \\
& voluntarios, que, son realizados por un miembro de la, \\
& organización, a su vez está observadas como contrarios a sus, \\
tendencias legitimas (Clegg \& Cooper, 2009). Entonces, estas \\
contraproducente \\
formas de comportamientos en el espacio de laboral intimidan \\
la, comodidad de la organización, por lo tanto, asimismo se \\
perjudican sus integrantes. \\
Por otro lado, Robinson \& Bennett explican, que los \\
comportamientos contraproducentes son aquellos procederes \\
antisociales manifestadas por los operarios ,a, la hora de \\
efectuar su trabajo, que son comportamientos negativos y \\
perjudiciales para la organización (Omar et al., 2012; \\
Koopmans et al., 2011).
\end{tabular}

Fuente: Elaborado en base a los autores

\section{Conclusiones}

Luego de hacer una descripción teórica acerca del desempeño laboral apoyándonos en el origen de la variable y sus respectiva dimensiones ,según el enfoque del modelo establecido por Linda Koopmans, llegando a la conclusión que es necesario el conocimiento acerca del desempeño laboral dentro de la organización ,debido a que es una fuente primordial para el desarrollo de esta misma, seguido el desempeño está asociado con las emociones o el pensar de las personas, de esta tenemos dimensiones importantes como el desempeño de las tareas que se visualiza como el empleado realiza su actividades dentro de la organización, en cambio el desempeño contextual revela como las personas tiene actitudes frente a situaciones ocasionales, por último los comportamientos contraproducentes que explica algunas conductas que optan los individuos a ciertas ocasiones en las que su reacción no es beneficiosa para la empresa a lo contrario, es negativa. Debido a lo mencionado previamente, podemos afirmar que esta investigación ofrece conocimientos teóricos para la formulación de textos de revisión o un análisis estadístico del desempeño laboral. 


\section{Referencias}

Aguirre, A., Castillo, A. M. y Tous, D. (1991). Administración de empresas. Málaga: Ed. Edinford.

Álamo, A., \& Marquez, A. (2012). Relación entre la calidad del intercambio lider-miembro y el síndrome de burnout en la fuerza de ventas [Universidad Católica Andrés Bello] http://w2.ucab.edu.ve/tl_files/escuela_ciencias_sociales/Trabajo\%20de\%20Grado/Tesis \%20Alamo\%20y\%20Marquez.pdf

Álvarez, B. , Porraspita, D. A., \& Indacochea, B. (2018). El desempeño laboral: un problema social de la ciencia. Didasc@ lia: Didáctica y Educación, 9(2), 147-158. https://dialnet.unirioja.es/servlet/articulo?codigo=6596591

Ana, C., Maria, L., \& Nora, R. (2011). El impacto de la rotación de personal en las empresas contructoras del estado de Nuevo León. Revista de la Facultad de Arquitectura de la Universidad Autónoma de Nuevo León, 5(2011), 83-91. https://www.redalyc.org/pdf/3536/353632025006.pdf

Araujo, M., \& Leal Guerra, M. (2007). Inteligencia emocional y desempeño laboral en las instituciones de educación superior públicas. CICAG: Revista del Centro de Investigación

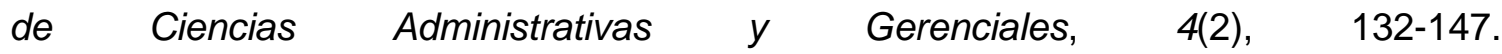
https://dialnet.unirioja.es/servlet/articulo?codigo=3218188

Argoti, E. (2020). Relación de la inteligencia emocional con el desempeño laboral. Ciencia \& Salud, 3(11), 41-46. http://revistas.usc.edu.co/index.php/CienciaySalud/article/view/505

Baltodano, G., \& Leyva, O. (2020). La productividad laboral : Una mirada a las necesidades de las Pymes en México. Revista Ciencia Jurídica y Política, 11(6), 15-30. https://portalderevistas.upoli.edu.ni/index.php/5revciencasjuridicasypoliticas/article/view/633

Bautista, R., Cienfuegos, R., \& Aquilar, J. (2020). El desempeño laboral desde una perspectiva teórica. Revista de Investigación Valor Agregado, 7(1), 54-60. https://doi.org/https://doi.org/10.17162/riva.v7i1.1417

Benavidez, O. (2002). Reseña del libro "Competencias y Competitividad". Diseño para Organizaciones Latinoamericanas" de Olga Benavides Espíndola. https://bit.ly/324Eu70

Beverly, D., Williams, J. A., \& Kitterlin, M. (2012). Leaders Exhibiting High Emotional Intelligence Are More Dedicated to Their Job Performance. Open Journal of Leadership, 01(04), 37-41. https://doi.org/10.4236/ojl.2012.14006

Bolzan, C. (2018). Valores personales, valores organizacionales y medio ambiente: una revisión teórica. Desenvolve Revista de Gestão do Unilasalle, 7(2), 75. https://doi.org/10.18316/desenv.v7i2.4415

Buitrón, S., \& Navarrete, P. (2008). El docente en el desarrollo de la inteligencia emocional: 
reflexiones y estrategias. Revista Digital de Investigación en Docencia Universitaria, 4(1), $1-8$.

Chavez, M. (2020). “Diseño y ejecución de un plan de comunicación de beneficios en una gran empresa del sector útiles escolares y de oficina: El caso de Continental-Standford [Universidad de Lima]. http://doi.org/10.26439/ulima.tesis/11313

Chiavenato, I. (2002). Gestión del Talento Humano. Mc Graw Hill: México. https://jgestiondeltalentohumano.files.wordpress.com/2013/11/gestion-del-talentohumano-idalberto-chiavenato-3th.pdf

Clegg, S. R., \& Cooper, C. L. (2009). The SAGE Handbook of Organizational Behavior: Volume II - Macro Approaches. SAGE Publications Ltd. https://doi.org/10.4135/9781849200455.

Drucker, P. F (1999). Los desafíos de la gerencia para el siglo XXI. Editorial Norma.

Espinoza, M., Sanhueza, O., Ramírez, N., \& Sáez, K. (2015). A validation of the construct and reliability of an emotional intelligence scale applied to nursing students. Revista LatinoAmericana de Enfermagem, 23(1), 139-147. https://doi.org/10.1590/01041169.3498.2535

Eufracio, B. (2018). Desempeño laboral y satisfacción del usuario en la empresa veterinaria San Mateo S.A.C. distrito de Carabayllo, año 2017. Universidad César Vallejo. https://hdl.handle.net/20.500.12692/15208

Forbes, R. (2013). La psicología organizacional positiva y la mejora organizacional. Exito Empresarial, $1(227)$, 1-3. http://www.cegesti.org/exitoempresarial/publicaciones/publicacion_227_040313_es.pdf

Fuentes, D., Chapis, E., \& Chapis, E. (2019). Administración de los recursos humanos. Universidad y Sociedad, 9(2), 313-318. http://scielo.sld.cu/pdf/rus/v11n3/2218-3620-rus11-03-186.pdf

Ribes, G., Perelló, M. R., \& Herrero, A. (2018). Dirección de recursos humanos. Gestión de personas. Colección Académica.

Hernández, E. (2008). Inteligencia emocional Y desempeño laboral de los supervisores de las industrias citrícolas de Montemorelos. https://es.scribd.com/document/308237253/TesisEstrella-Hernandez

Hernández, I., \& Vázquez, J. (2018). El supermultiplicador, la acumulación de capital, las exportaciones y el crecimiento económico. Super-Multiplier, Capital Accumulation, $\begin{array}{llll}\text { Exports and Economic } \quad \text { Growth., 85(338), } & \text { 411-432. }\end{array}$ https://doi.org/http://dx.doi.org/10.20430/ete.v85i338.542

Ivars Baidal, J. A., Solsona Monzonís, F. J., \& Giner Sánchez, D. (2016). Gestión turística y tecnologías de la información y la comunicación (TIC): El nuevo enfoque de los destinos $\begin{array}{llll}\text { inteligentes. Documents d'Anàlisi Geogràfica, } & \text { 62(2), }\end{array}$ https://doi.org/10.5565/rev/dag.285 
Koopmans, L. (2015). Assessing Job Performance Using Brief Self-report Scales: The Case of the Journal of Work and Organizational Psychology Assessing Job Performance Using Brief Self-report Scales: The Case of the. Journal of Work and Organizational Psychology, 35(1),

195-205. http://eds.b.ebscohost.com/eds/pdfviewer/pdfviewer?vid=10\&sid=29afb6fc-43e2-46ca9f9c-7100a1b72b22\%40sessionmgr103

Koopmans, L., Bernaards, C. M., Hildebrandt, V. H., Schaufeli, W. B., De Vet Henrica, C. W., \& Van Der Beek, A. J. (2011). Conceptual frameworks of individual work performance: A systematic review. Journal of Occupational and Environmental Medicine, 53(8), 856-866. https://doi.org/10.1097/JOM.0b013e318226a763

Lastini, S., \& Veliz, D. (2016). Feed back mejoramiento. Retroalimentación positiva para el mejoramiento del entrenamienton y liderazgo, 147, 11-40. http://recursosbiblio.url.edu.gt/tesisjcem/2016/05/43/Veliz-Dulce.pdf

Mamanil, Y., \& Avendaño, M. (2019). Desempeño laboral: una revisión teórica. https://repositorio.upeu.edu.pe/bitstream/handle/20.500.12840/2260/Yini_Trabajo_Bachi Ilerato_2019.pdf?sequence=1\&isAllowed=y

María, A., \& Pérez, S. (2011). La gestión universitaria y el clima organizacional. Educacion Medica Superior, 25(2), 164-177.

Martín, P. (2018). Teletrabajo y comercio electrónico [Madrid : Ministerio de Educación, Cultura y Deporte, 2018.]. Permalink: http://digital.casalini.it/9788436958379

Medina, S. (2017). Desempeño laboral del personal administrativo nombrado de la Municipalidad Provincial del Santa. Chimbote 2017. Universidad César Vallejo.

Mejía, F. (2010). Gestión escolar: un asunto de mercado, de Estado o de sociedad. Algunas experiencias internacionales. Revista Latinoamericana de Estudios Educativos (México), $X L(1), 9-52$. https://www.redalyc.org/pdf/270/27018883002.pdf

Moscoso, M. (2019). Hacia una integración de mindfulness e inteligencia emocional en psicología y educación. Liberabit: Revista Peruana de Psicología, 25(1), 107-117. https://doi.org/10.24265/liberabit.2019.v25n1.09

Navarro, V., Odena, M. C., Ackermann, M. A., \& Morales, E. (2016). Informe breve. Revista de la Facultad de Medicina de la UNNE, 36(2), 37-41.

Newstrom, J. (2000). Comportamiento Humano en el trabajo. Mc Graw Hill: México https://uachatec.com.mx/wpcontent/uploads/2019/11/Comportamiento_humano_en_el_trabajo.pdf

Omar, A. (2010). Comportamientos extra papel: de la colaboración a la transgresión. Revista $\begin{array}{llll}\text { Interamericana de Psicologia } & \text { 32-53. }\end{array}$ http://revista.cincel.com.co/index.php/RPO/article/view/103

Omar, A., Vaamonde, J. D., \& Delgado, H. U. (2012). Comportamientos contraproducentes en 
el trabajo: diseño y validación de una escala* Counterproductive Work Behavior: Design and Validation of a Scale. Perspectivas en Psicología, 8(2), 249-265. http://www.redalyc.org/pdf/679/67925837004.pdf

Ortega, C. (2015). Las competencias laborales y el clima organizacional del personal administrativo en las Universidades Tecnológicas Privadas del Lima [Universidad Nacional Mayor de San Marcos]. https://hdl.handle.net/20.500.12672/4308

Pacheco, N. E., \& Fernández-berrocal, P. (2004). El papel de la inteligencia emocional en el alumnado : evidencias empíricas. Revista Electronica De Investigacion Educativa, 6(2), $1-17$. http://www.scielo.org.mx/scielo.php?script=sci_arttext\&pid=S160740412004000200005

Palací, F. J. (2005). Psicología de la Organización. Pearson de la Educación: Madrid

Pastor, A. (2018). Clima y desempeño laboral en trabajadores administrativos de una empresa privada de combustibles e hidrocarburos, Lima 2017. Universidad San Ignacio de Loyola. http://repositorio.usil.edu.pe/handle/USIL/3466

Pedraza, E., Amaya, G., \& Conde, M. (2010). Desempeño laboral y estabilidad del personal administrativo contratado de la Facultad de Medicina de la Universidad del Zulia. Revista de Ciencias Sociales, 16(3), 493-505. https://doi.org/10.31876/rcs.v16i3.25519

Pegoraro, F. V. (2009). Relación entre inteligencia emocional y desempeño Contextual (Caso de estudio). Universidad Católica Andrés Bello. http://biblioteca2.ucab.edu.ve/anexos/biblioteca/marc/texto/AAR7038.pdf

Robbins, S. P. y Judge, T. A. (2009). Comportamiento Organizacional (13 Ed.) Pearson: México

https://frrq.cvg.utn.edu.ar/pluginfile.php/15550/mod_resource/content/0/ROBBINS comportamiento-organizacional-13a-ed-_nodrm.pdf

Robbins, S. P. y Judge, T. A. (2013). Comportamiento Organizacional (15 Ed.) Pearson: México.

http://chamilo.cut.edu.mx:8080/chamilo/courses/psicologiaorganizacionaloriente2019/do cument/comportamiento_organizacional_15edi-robbins.pdf

Rodríguez-Marulanda, K. P., \& Lechuga-Cardozo, J. I. (2020). Desempeño laboral de los docentes de la Institución Universitaria ITSA. Revista Escuela de Administración de Negocios, 87, 79-101. https://doi.org/10.21158/01208160.n87.2019.2452

Rosas-reyes, J. G., \& Roberto, E. (2015). Influencia de la inteligencia emocional en el desempeño laboral. Sapienza Organizacional, O(3), 53-68. https://www.redalyc.org/pdf/5530/553056601004.pdf

Rotundo, M. (2002). The relative importance of task, citizenship, and counterproductive performance to global ratings of job performance: a policy-capturing approach. The Journal of applied psychology, 87(1), 66-80. https://doi.org/10.1037/0021-9010.87.1.66 
Salanova, M., Martínez, I. M., \& Llorens, S. (2014). Una mirada más «positiva» a la salud ocupacional desde la psicología organizacional positiva en tiempos de crisis: Aportaciones desde el equipo de investigación wont. Papeles del Psicologo, 35(1), 2230.

http://repositori.uji.es/xmlui/bitstream/handle/10234/120543/ID62477.pdf?sequence=1\&i sAllowed =y

Sanders, K., Dorenbosch, L., \& De Reuver, R. (2008). The impact of individual and shared employee perceptions of HRM on affective commitment: Considering climate strength. Personnel Review, 37(4), 412-425. https://doi.org/10.1108/00483480810877589

Sladogna, M. (2017). Productividad- Definiciones y perspectivas para la negociacion colectiva. Journal of Chemical Information and Modeling, 53(9), 1689-1699. http://www.relats.org/documentos/ORGSladogna2.pdf

Topa, G., \& Morales, F. (2006). Identificación organizacional y proactividad personal en grupos de trabajo: un modelo de ecuaciones estructurales. Anales de Psicología / Annals of Psychology, 22(2), 234-242.

Torres, E., Fretel Quiroz, N. M., Coral Cevillano, M., \& Ramírez Chumbe, I. (2021). Inteligencia emocional y desempeño laboral de los profesionales de la salud de un hospital de Pucallpa. Revista Vive, 4(10), 64-71. https://doi.org/10.33996/revistavive.v4i10.76

Vercelli, A., \& Thomas, H. (2008). Repensando los bienes comunes: análisis socio-técnico sobre la construcción y regulación de los bienes comunes. Scientiae Studia. https://doi.org/10.1590/S1678-31662008000300010

Wolfe, F., Smythe, H. A., Yunus, M. B., Bennett, R. M., Bombardier, C., Goldenberg, D. L., Tugwell, P., Campbell, S. M., Abeles, M., Clark, P., Fam, A. G., Farber, S. J., Fiechtner, J. J., Michael Franklin, C., Gatter, R. A., Hamaty, D., Lessard, J., Lichtbroun, A. S., Masi, A. T., ... Sheon, R. P. (1990). The american college of rheumatology 1990 criteria for the classification of fibromyalgia. Arthritis \& Rheumatism, 33(2), 160-172. https://doi.org/10.1002/art.1780330203

Wu, J., \& Liu, A. M. M. (2006). A study on the relationship between organizational commitment and task performance in Chinese construction firms. Association of Researchers in Construction Management, ARCOM 2006 - Procs 22nd Annual ARCOM Conference, 1(September), 207-217. 